\title{
Efficacy of Omliv ${ }^{T M^{*}}$ an indigenous polyherbal formulation in the management of gallbladder/biliary sludge diseases
}

\begin{abstract}
Biliary sludge is thick concretions or precipitates like substance present in gallbladder and is considered as precursor of the gallstones. In recent era, cases have been increasing significantly because of the routine ultrasonography examination of the abdomen in any abdominal problem. Therefore, asymptomatic incidental capture of the sludge in gallbladder is common now. Most patients undergo operations due to fear of biliary complications. Omliv ${ }^{\mathrm{TM}}$, one tablet twice daily for 45 days has been tried in these patients with acceptable results. Gallbladder sludge has been washed out by this regime. Even nonmeasurable small concretions were also washed off. I present case series of three cases of different age groups to establish the possibility of the conservative management with $\mathrm{Omliv}^{\mathrm{TM}}$ in gallbladder/biliary sludge cases. The main objective of the present case report is the successful implication of the nonsurgical conservative approach to this subset of patients in whom the Omliv ${ }^{\mathrm{TM}}$ tablet has not only proved as economic mode of treatment but can avoid surgery also.
\end{abstract}

Keywords: gallbladder sludge, biliary sludge, cholecystectomy, laparoscopic cholecystectomy, Omliv ${ }^{\mathrm{TM}}$, conservative management, gallstone, ayurveda, herbal drug
Volume 12 Issue 2 - 2021

\section{Pankaj Srivastava}

Department of Surgery, Om Surgical Center \& Maternity Home, India

Correspondence: Dr. Pankaj Srivastava, Laparoscopic, Thoracic, Thoracoscopic \& VATS Surgeon Om Surgical Center \& Maternity Home, SA 17/3, P-4, Sri Krishna Nagar, Paharia, Ghazipur Road,Varanasi, UP, India, Tel +91-542-2586I91, Emaildrpankajbns@gmail.com

Received: April 15, 2021 | Published: April 23, 2021

\section{Introduction}

Biliary sludge was first described in 1970s with the advent of ultrasonography. Gallbladder sludge appears as low amplitude homogeneous echoes, layering on the posterior wall, and frequently forming fluid-fluid level with anechoic bile above it. It moves slowly with changes in patient position and sludge does not cause shadowing unless associated with gallstones. ${ }^{1}$ Sludge can get compacted forming a mass-like lesion referred to as tumefactive sludge. ${ }^{2}$ Biliary sludge is most commonly composed of classical solid plate-like cholesterol monohydrate crystals, as well as varying sizes of aggregated liquid crystals, calcium bilirubinate granules, and other calcium salts, most of which are embedded in strands of mucin gel produced by the gallbladder. $^{3-5}$

As such biliary sludge is considered as precursor of gallstones; therefore, treatment of biliary sludge is also similar to gallstone diseases. In asymptomatic and uncomplicated patients if biliary sludge is an incidental finding on ultrasonography done for other reasons, patient must be offered expectant treatment with followup ultrasonographic scans. Patients presented with complications or severe symptoms must be treated accordingly. Cholecystectomy is the definitive and gold standard treatment for symptomatic biliary sludge. In patients with pancreatitis or cholangitis who are not amenable for surgery, sphincterotomy is a reasonable alternative to cholecystectomy. ${ }^{6}$

Omliv $^{\mathrm{TM}}$ (Table 1) a polyherbal formulation contains ten different herbal plant products, was given to all the three patients and its efficacy was evaluated by the absence of sludge and/or concretions in the gallbladder after complete regimen. It was hypothesized that the Omliv ${ }^{\mathrm{TM}}$, a polyherbal formulation will completely clear the gallbladder lumen with the sludge and the non-measurable tiny microlithiasis in the treatment period of forty-five (45) days.

Table I Ingredients of Tablet Omliv ${ }^{T M}$

\begin{tabular}{llll}
\hline S.No. & Latin Name & Part Used & Quantity (Milligram)/Tablet \\
\hline I. & Andrographis paniculata & Whole plant & 50 \\
2. & Boerhaavia diffusa & Root & 50 \\
3. & Terminalia chebula & Fruit & 40 \\
4. & Terminalia bellirica & Fruit & 20 \\
5. & Phyllanthus emblica & Dry Fruits & 50 \\
6. & Adhatoda vasica & Green Leaves & 40 \\
7. & Tinospora cordifolia & Green Stem & 50 \\
8. & Azadirachta indica & Stem Bark & 20 \\
9. & Picrorhiza kurroa & Rhizome & 20 \\
10. & Swertia chirata & Whole Plant & 20 \\
II. & Excipients & - & Q.S. \\
\hline
\end{tabular}




\section{Case presentation}

\section{Case I}

A 40-years-old non-smoker, non-alcoholic, vegetarian male presented with mild to moderate pain in right upper abdomen for 4 to 5 months. Pain was aggravated after the meal. There was history of two episodes of vomiting after attack of severe pain in last one month. He consulted to his family physician for the same complaints and took antacids and proton pump inhibitors (PPIs) but got only time being relief. Ultrasonography (USG) abdomen Figures 1 \& 2 showed well distended gallbladder with normal wall thickness but sludge in the lumen. The patient was advised for the laparoscopic cholecystectomy and for the same referred to us. The patient was then thoroughly examined and nothing significant could be detected in systemic or local examination. The blood biochemistry and serology were also within normal limit except raised Aspartate transaminase (AST) $89 \mathrm{IU} / \mathrm{L}$ and Alanine aminotransferase (ALT) $142 \mathrm{IU} / \mathrm{L}$. Patient was given Omliv ${ }^{\mathrm{TM}}$, one tablet twice daily for 45 days. The follow-up USG (Figure 3 ) revealed normally distended gallbladder with normal wall thickness and clear lumen. It was concluded that the Omliv ${ }^{\mathrm{TM}}$ can clear the gallbladder sludge and is also effective for the symptomatic improvement of the patient.

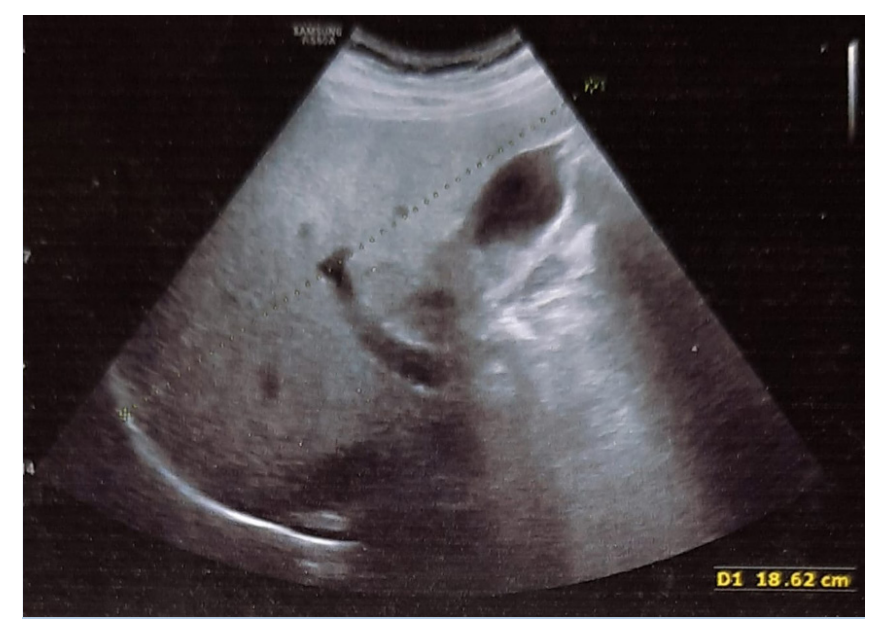

Figure I Case No. I [Pre-treatment ultrasonography scan showing biliary sludge].

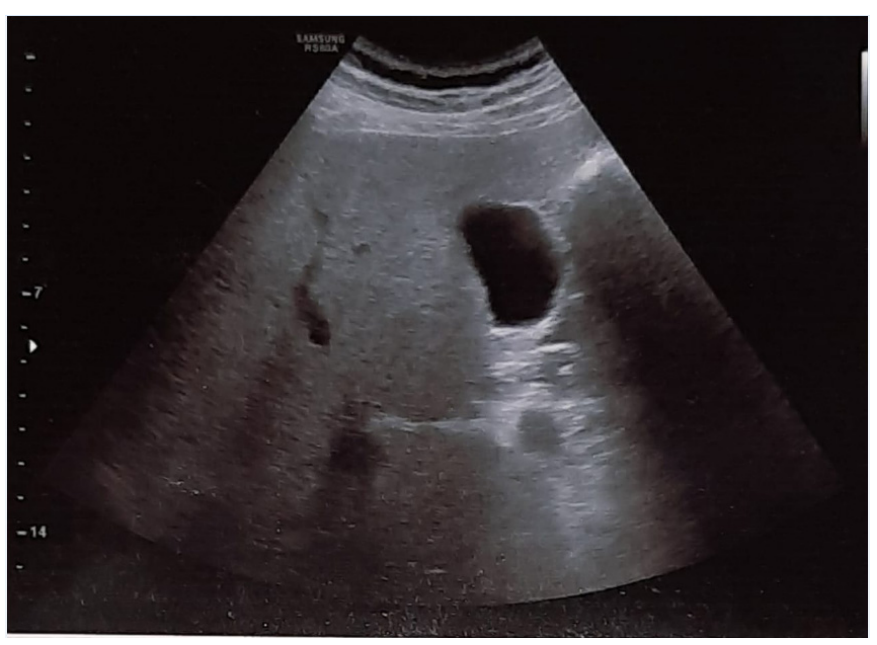

Figure 2 Case No. I [Pre-treatment ultrasonography scan showing biliary sludge].

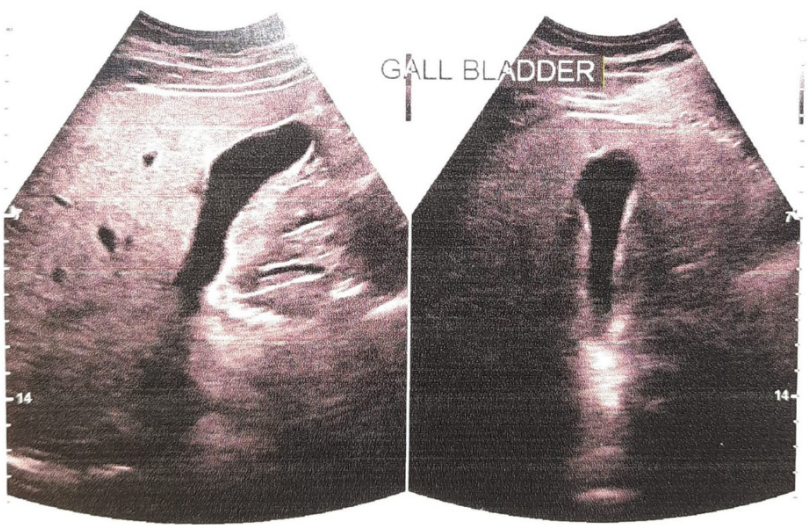

Figure 3 Case No. I [Post-treatment ultrasonography scan showing clear gallbladder lumen].

\section{Case 2}

A 35-years-old non-smoker, non-alcoholic, non-vegetarian, menstruating female presented with moderate to severe spasmodic pain in right upper abdomen and epigastric region for one year. Pain was aggravated after the fatty and spicy meal. There was history of several episodes of vomiting during attack of severe pain in last three month which occurred typically in night after 2-3hours of heavy meal. Vomiting was bilious in nature. She took antacids, H2 blockers, proton pump inhibitors and antiemetic along with pain killers but could not be improved. She had a history of hospital admission for once six months before. The patient was referred to us for the laparoscopic cholecystectomy. The patient was then thoroughly examined and nothing significant could be detected in systemic but on local examination, hepatomegaly was found with palpable gallbladder. Ultrasonography (USG) abdomen Figure 4 showed large over-distended gallbladder with mild wall thickness and thick sludge and concretions in the lumen. The blood biochemistry revealed high total leukocyte counts (14800/cumm) with neutrophilia (82\%). Serology showed raised Alkaline phosphatase (325 U/L), Aspartate transaminase (AST) $89 \mathrm{IU} / \mathrm{L}$ and Alanine aminotransferase (ALT) 142 IU/L. Rest of the lab parameters were within normal limits. Patient was given $\mathrm{Omliv}^{\mathrm{TM}}$, one tablet twice daily along with broad-spectrum antibiotics and PPIs. Antibiotics and PPIs were stopped after 7days when TLC became normal (7300/cumm) and the Omliv ${ }^{\mathrm{TM}}$, one tablet twice daily continued for the complete 45 days therapy. The follow-up USG (Figure 5) revealed normally distended gallbladder with normal wall thickness and clear lumen. There was no evidence of sludge or concretions in the gallbladder lumen. Blood biochemistry and abnormal liver function tests also come within normal limits.

\section{Case 3}

A 14-years-old vegetarian female child presented with mild to moderate pain in whole of the abdomen for one year. Pain was not related to the meal. There was history of few episodes of vomiting after attack of moderate to severe pain in last three months. She attained her menarche at 12 years of age. She consulted to pediatric surgeon who gave her antispasmodic and antacids. Ultrasonography (USG) abdomen (Figure 6) showed well distended gallbladder with normal wall thickness but few small concretions and sludge in the lumen. The pediatric surgeon advised her laparoscopic cholecystectomy. She consulted me to the second opinion for the same ailment. The systemic and local examinations were within normal limits. The blood biochemistry and serology were also within normal limit. Because 
there was no obvious complication and problem, patient was given a chance for the conservative management before the definitive treatment i.e. Cholecystectomy. Patient was given Omliv ${ }^{\mathrm{TM}}$ one tablet twice daily and Tablet Drotaverine $20 \mathrm{mg}$ SOS for 45 days. The follow-up USG (Figure 7) revealed normally distended gallbladder with normal wall thickness and clear lumen. It was concluded that the OmlivTm can clear the gallbladder sludge and is also effective for the symptomatic improvement of the patient.

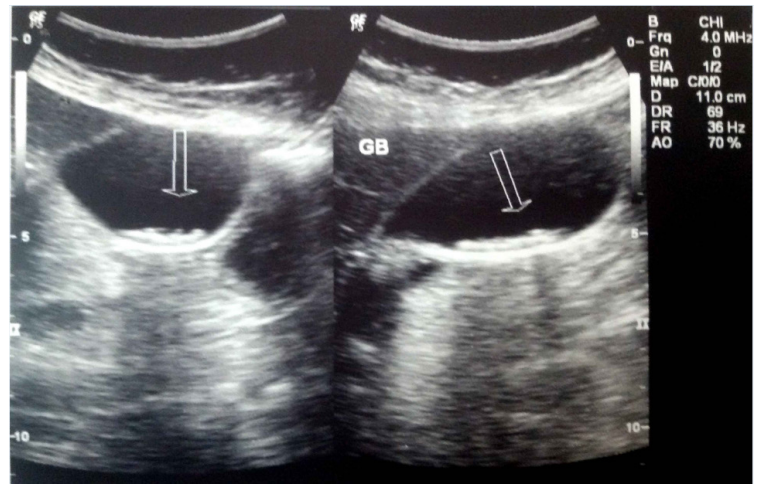

Figure 4 Case No. 2 [Pre-treatment ultrasonography scan showing biliary sludge \& concretions]

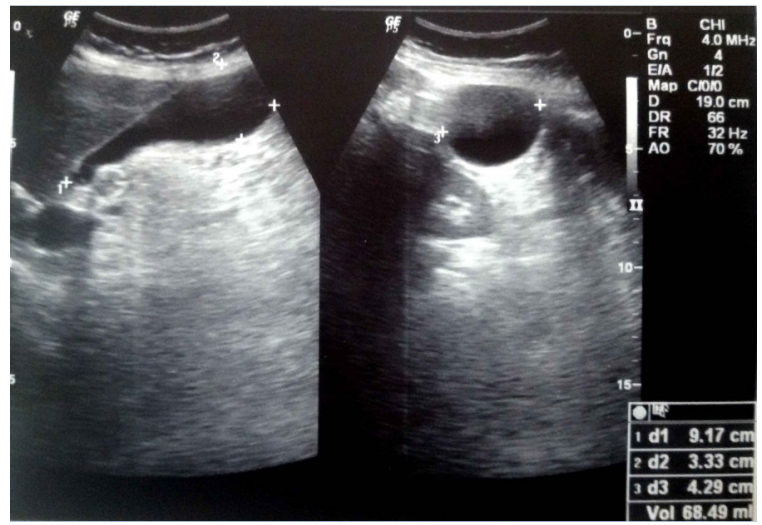

Figure 5 Case No. 2 [Post-treatment ultrasonography scan showing clear gallbladder lumen].

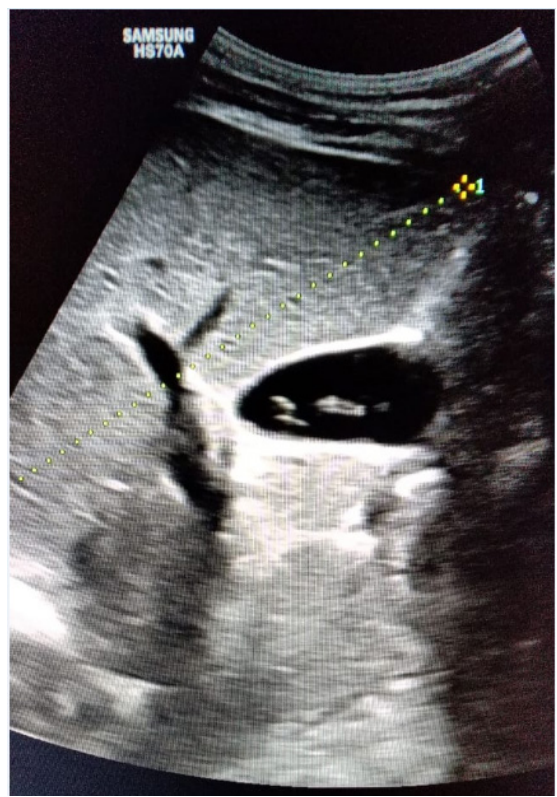

Figure 6 Case No. 3 [Pre-treatment ultrasonography scan showing biliary sludge].

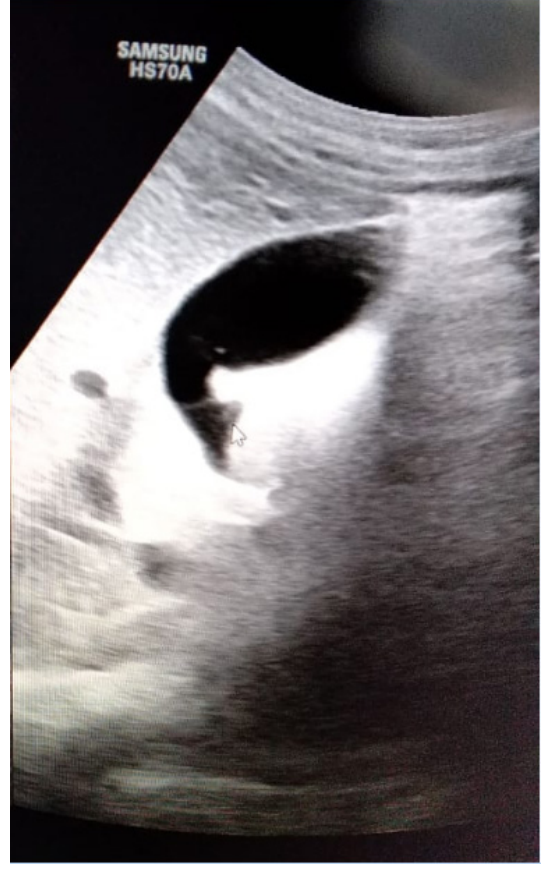

Figure 7 Case No. 3 [Post-treatment ultrasonography scan showing clear gallbladder lumen].

\section{Discussion}

The pathogenesis of biliary sludge is thought to be the same as for gallstones, with sludge functioning as a precursor to stone formation. ${ }^{7}$ A combination of impaired gallbladder motility and alteration in nucleation factors leads to the formation of sludge with additional precipitate aggregation resulting in gallstone formation. ${ }^{8}$ The results from the Lee's study indicate that in patients with sludge the gallbladder is abnormal, showing mucus hypersecretion and glandular metaplasia. This leads to an increase in the mucus content of gallbladder bile, which in turn may result in nucleation of cholesterol crystals and may be regarded as the embryonic stage of gallstone disease. ${ }^{9}$ Clinical conditions and events associated with the formation of biliary sludge include rapid weight loss; pregnancy; chronic illness include AIDS, cirrhosis, sickle cell anemia; acute illness include intensive care therapy, surgery, spinal cord injury; drug therapy include ceftriaxone, cyclosporine and octreotide, and bone marrow or solid organ transplantation. ${ }^{6}$ In one natural history study covering a 3-year period, sludge spontaneously disappeared in $50 \%$ of patients and remained asymptomatic in another $20 \% ; 5 \%$ to $15 \%$ of patients developed gallstones and $10 \%$ to $15 \%$ of patients developed symptoms. ${ }^{10}$

Biliary sludge is usually diagnosed by transabdominal ultrasound but new ultrasound scanners employing second harmonic imaging may lead to improved detection of sludge. ${ }^{11}$ According to several authors, the upper limit for normality of the gallbladder wall thickness is $3 \mathrm{~mm} .{ }^{12,13}$ However, in patients under inappropriate fasting, the parietal thickness may exceed such a limit because of the organ's smooth muscle contraction. ${ }^{14}$ So, 8 -hour fasting before the examination is recommended, particularly in cases where the gallbladder is the focus of the study. Gallbladder wall thickening is classified as mild (between 4 and $7 \mathrm{~mm}$ ), marked $(>7 \mathrm{~mm})$, and in focal or diffuse. ${ }^{15}$ The gallbladder wall thickness had been evaluated according to the aforementioned criteria. ${ }^{15}$

Laparoscopic cholecystectomy remains the gold standard treatment for the symptomatic cases and those who have associated 
complications. Since most of the patients with gallbladder sludge are asymptomatic and has been diagnosed to have the sludge in their gallbladder due to routine ultrasonography of abdomen for other reasons ${ }^{16}$ conservative management may be offered to them for the expectant treatment. In the present case report all the three cases were symptomatic but could not be labeled as acute or chronic cholecystitis based on examination and other ancillary investigations. Contrary to the previous belief that symptomatic cases should be offered cholecystectomy as the treatment of choice, conservative treatment with the Omliv ${ }^{\mathrm{TM}}$ had been tried very cautiously with close follow-up that ended up with excellent results not only in terms of alleviation of the symptoms but also with the disappearance of the gallbladder sludge.

Herbal cholagogues target different components of the complex bile production and secretion system, and exert their action via diverse routes, such as cholecystokinin-dependent and independent gallbladder contraction, up-regulation of the bile acid synthesis, stimulation of the bile salt export pump, multidrug resistance protein transporter system, and osmotic bile flow. ${ }^{17}$

Since 2017, we have been trying one established regimen named as "Srivastava regimen for biliary sludge" which contains oral ampicillin, UDCA and Phaltrikadi Kvatha (decoction) or Ghanbati (Tablet) for minimum of 45 days, dietary fat restriction, plenty of water intake and avoiding all the possible confounding factors of gallstone diseases. The results have been very promising and acceptable. The Omliv ${ }^{\mathrm{TM}}$ has been later developed after extensive research in Phaltrikadi Tablet as mono drug therapy. That's why the oral ampicillin and UDCA have been dropped in current treatment protocol. This conservative approach not only saves the surgical procedure on patients but the huge hospital expenses also.

\section{Conclusion}

It was concluded that the $\mathrm{Omliv}^{\mathrm{TM}}$ can not only clear the gallbladder sludge (Case 1-3) but very tiny concretions also as seen in Case 2 of our series. The drug is also proved effective in alleviating the dyspeptic symptoms associated with the biliary disorders. More data is required to establish $\mathrm{Omliv}^{\mathrm{TM}}$ as a definite treatment drug in larger perspective as far as the surgical burden and economic loss are concern. Studies are also required to evaluate the exact mechanism of action of the herbal drugs. Though in the formulation of many drugs it is very difficult to conclude inference on the role of each drug but as a formulation evaluation at molecular level will give more insight. The current case series throws a ray of hope in the dark mist of biliary ailments.

\section{Acknowledgments}

None.

\section{Conflicts of interest}

Authors declare that we have no conflicting interests.

\section{Funding}

The authors did not receive any grant for this research study.

\section{References}

1. Ferreira AC, Filho FM, Mauad FM et al. Clinical and ultrasonographic risk factors associated with asymptomatic gallstones in wome). Radiol Bras. 2004;37(2):77-82 .

2. Chatterjee A, Vendrami CL, Nikolaidis P. Uncommon Intraluminal Tumors of the Gallbladder and Biliary Tract: Spectrum of Imaging Appearances. RadioGraphics. 2019;39(2):388-412.

3. Lee SP, Nicholls JF. Nature and composition of biliary sludge. Gastroenterology. 1986;90(3):677-686.

4. Lee SP, Maher K, Nicholls JF. Origin and fate of biliary sludge. Gastroenterology. 1988;94(1):170-176.

5. Angelico M, De Santis A, Capocaccia L. Biliary sludge: A critical update. J Clin Gastroenterol. 1990;12(6):656-662.

6. https://pubmed.ncbi.nlm.nih.gov/15010024/

7. Srivastava P, Srivastava S, Srivastava R. Biliary sludge: can we avoid punishing gallbladder by Srivastava regimen of integrated approach of medicine? J Liver Res Disord Ther. 2017;3(5):119-121.

8. Conrad MR, Janes JO, Dietchy J. Significance of low level echoes within the gallbladder. AJR Am J Roentgenol. 1979;132(6):967-972.

9. Johnston DE, Kaplan MM. Pathogenesis and treatment of gallstones. $N$ Engl J Med. 1993;328(6):412-421.

10. Gregor JC, Ponich TP, Detsky AS. Should ERCP be routine after an episode of "idiopathic" pancreatitis? A cost-utility analysis. Gastrointest Endosc. 1996;44(2):118-123.

11. Lee SP, Hayashi A, Kim YS. Biliary sludge: curiosity or culprit? Hepatology. 1994;20(2):523-525.

12. Levy AD, Murakata LA, Abbott RM, et al. From the archives of the AFIP: Benign tumors and tumor like lesions of the gallbladder and extrahepatic bile ducts: radiologic-pathologic correlation. Radiographics. 2002;22(2):387-413.

13. Wibbenmeyer LA, Sharafuddin MJ, Wolverson MK, et al. Sonographic diagnosis of unsuspected gallbladder cancer: imaging findings in comparison with benign gallbladder conditions. AJR Am J Roentgenol. 1995;165(5):1169-1174.

14. AIUM practice guideline for the performance of an ultrasound examination of the abdomen and/ or retroperitoneum. J Ultrasound Med. 2008;27:319-26.

15. Barbosa ABR, souza, LRMFD, pereira RS, et al. Gallbladder wall thickening at ultrasonography: how to interpret it? Radiol Bras. 2011.44(6).

16. Pazzi P, Gamberini S, Buldrini P, et al. Biliary sludge: the sluggish gallbladder. Dig Liver Dis. 2003;35(Suppl 3):S39-S45.

17. Spiridonov NA. Mechanisms of Action of Herbal Cholagogues. Med Aromat Plants. 2012;1:107. 